\title{
Temporal percolation in activity-driven networks
}

\author{
Michele Starnini and Romualdo Pastor-Satorras \\ Departament de Física i Enginyeria Nuclear, Universitat Politècnica de Catalunya, Campus Nord B4, 08034 Barcelona, Spain
}

(Received 18 December 2013; published 14 March 2014)

\begin{abstract}
We study the temporal percolation properties of temporal networks by taking as a representative example the recently proposed activity-driven-network model [N. Perra et al., Sci. Rep. 2, 469 (2012)]. Building upon an analytical framework based on a mapping to hidden variables networks, we provide expressions for the percolation time $T_{p}$ marking the onset of a giant connected component in the integrated network. In particular, we consider both the generating function formalism, valid for degree-uncorrelated networks, and the general case of networks with degree correlations. We discuss the different limits of the two approaches, indicating the parameter regions where the correlated threshold collapses onto the uncorrelated case. Our analytical predictions are confirmed by numerical simulations of the model. The temporal percolation concept can be fruitfully applied to study epidemic spreading on temporal networks. We show in particular how the susceptible-infected-removed model on an activity-driven network can be mapped to the percolation problem up to a time given by the spreading rate of the epidemic process. This mapping allows us to obtain additional information on this process, not available for previous approaches.
\end{abstract}

DOI: 10.1103/PhysRevE.89.032807

PACS number(s): $89.75 . \mathrm{Hc}, 05.40 . \mathrm{Fb}$

\section{INTRODUCTION}

The traditional approach of network science [1] to the study of complex interacting systems is based in a mapping to a static network or graph, in which nodes represent interacting units and edges, standing for pairwise interactions, are fixed and never change in time. This approach has been proven very powerful, providing a unified framework to understand the structure and function of networked systems [2] and to unravel the coupling of a complex topology with dynamical processes developing on top of it [3,4]. Many networks, however, are not static, but have instead an evolving topology, with connections appearing and disappearing with some characteristic time scales. A static approximation is still valid when such time scales are sufficiently large, such as in the case of the Internet [5]. In other cases, however, this approximation is incorrect. This is particularly evident in the case of social interactions networks, which are formed by a sequence of contact or communication events, lasting a certain amount of time, constantly being created and terminated between pairs of individuals. The recent availability of large amounts of data on social networks, such as mobile phone communications [6], face-to-face social interactions [7], or large scientific collaboration databases [8], has spurred the interest in the temporal dimension of social networks, leading to the development of new tools and concepts embodied in the new theory of temporal networks [9]. Key results of these efforts have been the observation of the "bursty", heterogeneous patterns of social contacts, revealed by distributions of the time of contact between pairs of individuals, the total time of contact for an individual, or the gap times between consecutive interactions involving the same individual, showing a heavy tailed form [6,7,10-13]. The alteration of available edges, and their rate of appearance, also has important effects on dynamical processes running on top of temporal networks [14-24].

While the exact characterization of a temporal network is given by the full ordered sequence of contacts (edges) present in it at time $t$ [9], this information is not always easily available. Thus, sometimes one only has access to coarse-grained information, in terms of an integrated network, constructed by integrating the temporal information up to a time $T$, in such a way that we consider the existence of an edge between nodes $i$ and $j$ in the integrated version if there was ever an edge in the contact sequence at any time $t \leqslant T$. While these integrated representations for a fixed (in general large) time have been since long a useful device to understand network properties [25], less is known about the effect of the integration time $T$ in the structural properties of the integrated network, an issue which has been recently shown to have relevant consequences for dynamical processes [26]. In this context, a particularly important piece of information is the connectivity properties of the integrated network as a function of time, and in particular the birth and evolution of a giant component. Indeed, at a given instant of time $t$, a temporal network can be represented by a single network snapshot, which is usually very sparse, composed of isolated edges, stars, or cliques. As we integrate more and more of those snapshots, the integrated network will grow, until at some time $T_{p}$ it will percolate; i.e., it will possess a giant connected component with a size proportional to the total number of individuals in the network. The time of the first appearance of this giant component is not only an important topological property of integrated networks, but it is also relevant for the evolution of dynamical processes, in the sense that any process with a characteristic lifetime $\tau<T_{p}$ will be unable to explore a sizable fraction of the network.

Here we will consider the temporal percolation properties of the integrated form of temporal networks. In order to be able to perform an analytical study, we will focus on the recently proposed activity-driven-network model [8], a temporal network model built on the observation that the establishment of social interactions is driven by the activity of individuals, urging them, with different levels of intensity, to interact with their peers. Building on the mapping of the integrated network ensuing from this model into the class of network models with hidden variables [27,28], we compute analytic expressions for the percolation time and the size of the giant component of the integrated network. An added 
value of our approach is the possibility to extend the mapping of epidemic spreading into percolation processes in static networks [29] into the temporal case. Thus our results can be extended to provide the epidemic threshold and the outbreak size of the susceptible-infected-removed epidemic model [30].

\section{THE ACTIVITY-DRIVEN MODEL: DEFINITION AND TOPOLOGICAL PROPERTIES}

The activity-driven network model [8] is defined in terms of $N$ individuals (agents), each one endowed with an activity $a_{i}$, defined as the probability that she starts a social interaction with other agents per unit time. The activity of the agents is a random variable, extracted from the activity potential distribution $F(a)$. Focusing on the emergence of the integrated network, dynamical creation of links follows an asynchronous scheme [28]: Every time step $\Delta t=1 / N$, an agent $i$ is chosen uniformly at random. With probability $a_{i}$, the agent becomes active and generates $m$ links that are connected to $m$ randomly chosen agents. These links last for a period of time $\Delta t$ (i.e., are erased at the next time step). Finally, time is updated $t \rightarrow t+\Delta t$.

The topological properties of the integrated activity-driven network at time $T$ have been studied in Ref. [28] by means of a mapping to a hidden variables network model [27], which is based on the connection probability $\Pi_{T}\left(a, a^{\prime}\right)$ that two nodes with activity $a$ and $a^{\prime}$ are connected in the integrated network at time $T$. For large $N$, this connection probability takes the form [28]

$$
\Pi_{T}\left(a, a^{\prime}\right)=1-\exp \left[-\lambda\left(a+a^{\prime}\right)\right],
$$

where $\lambda=T / N$ and we have set $m=1$ to simplify calculations. The topological properties of the integrated network are encoded in the propagator $g_{T}(k \mid a)$, defined as the probability that a node with activity $a$ has integrated degree $k$ at time $T$, and whose generating function $\hat{g}_{T}(z \mid a)=\sum_{k} g_{T}(k \mid a) z^{k}$ satisfies the general equation $[27,28]$

$$
\ln \hat{g}_{T}(z \mid a)=N \sum_{a^{\prime}} F\left(a^{\prime}\right) \ln \left[1-(1-z) \Pi_{T}\left(a, a^{\prime}\right)\right] .
$$

From the propagator, the degree distribution of the integrated network at time $T$ is trivially given by

$$
P_{T}(k)=\sum_{a} F(a) g_{T}(k \mid a) .
$$

In the limit of small $\lambda$ or $N \gg T$ with constant $T$, which we assume from now on, the connection probability Eq. (1) can be approximated as

$$
\Pi_{T}\left(a, a^{\prime}\right) \simeq \lambda\left(a+a^{\prime}\right) .
$$

In this same limit $\lambda \rightarrow 0$, Eq. (2) can be solved, leading to a propagator with the form of a Poisson distribution with mean $T(a+\langle a\rangle)$. From it, we obtain an asymptotic degree distribution [28]

$$
P_{T}(k) \simeq \frac{1}{T} F\left(\frac{k}{T}-\langle a\rangle\right) .
$$

Equation (5) encloses a fundamental property of the activitydriven model, since it shows that the degree distribution of the time-integrated network has the same functional form of the injected activity potential distribution, which can be measured in real-world social networks. This and other topological properties, concerning degree correlations and clustering spectrum, have been thoroughly described in Ref. [28].

\section{GENERATING FUNCTION APPROACH TO PERCOLATION}

Percolation in random networks can be studied applying the generating function approach developed in Ref. [31], which is valid assuming the networks are degree uncorrelated. Let us define $G_{0}(z)$ and $G_{1}(z)$ as the degree distribution and the excess degree distribution (at time $T$ ) generating functions, respectively, given by [2]

$$
G_{0}(z)=\sum_{k} P_{T}(k) z^{k}, \quad G_{1}(z)=\frac{G_{0}^{\prime}(z)}{G_{0}^{\prime}(1)} .
$$

The size of the giant connected component, $S$, is then given by

$$
S=1-G_{0}(u)
$$

where $u$, the probability that a randomly chosen vertex is not connected to the giant component, satisfies the self-consistent equation

$$
u=G_{1}(u) .
$$

The position of the percolation threshold can be simply obtained by considering that $u=1$ is always a solution of Eq. (8), corresponding to the lack of giant component. A physical solution $u<1$, corresponding to a macroscopic giant component, can only take place whenever $G_{1}^{\prime}(1)>1$, which leads to the Molloy-Reed criterion [32]:

$$
\frac{\left\langle k^{2}\right\rangle_{T}}{\langle k\rangle_{T}}>2,
$$

where $\left\langle k^{n}\right\rangle_{T}=\sum_{k} k^{n} P_{T}(k)$ is the $n$th moment of the degree distribution at time $T$.

In the case of the activity-driven model, these moments can be computed noticing, from Eq. (3), that $\left\langle k^{n}\right\rangle_{T}=$ $\sum_{a} F(a) \sum_{k} k^{n} g_{T}(k \mid a)$. Since the propagator has the form of a Poisson distribution, the moments of the degree distribution simply read as

$$
\left\langle k^{n}\right\rangle_{T}=\sum_{m=1}^{n}\left\{\begin{array}{l}
n \\
m
\end{array}\right\} T^{m} \kappa_{m},
$$

where $\left\{\begin{array}{l}n \\ m\end{array}\right\}$ are the Stirling numbers of the second kind [33] and

$$
\kappa_{m}=\sum_{a} F(a)(a+\langle a\rangle)^{m}=\sum_{i=0}^{m}\left(\begin{array}{c}
m \\
i
\end{array}\right)\left\langle a^{i}\right\rangle\langle a\rangle^{m-i} .
$$

The ratio $\left\langle k^{2}\right\rangle_{T} /\langle k\rangle_{T}$ is a monotonic, growing function of $T$, and it will fulfill the condition Eq. (9) for $T>T_{p}^{0}$, defining a percolation time

$$
T_{p}^{0}=\frac{\kappa_{1}}{\kappa_{2}}=\frac{2\langle a\rangle}{\left\langle a^{2}\right\rangle+3\langle a\rangle^{2}} .
$$

This percolation time is independent of $N$, and thus guarantees the fulfilment of the condition $\lambda \ll 1$ assumed in the derivation of Eq. (4). We can obtain information on the size of the giant 
component $S$ for $T>T_{p}^{0}$ from Eqs. (6) and (3), using the Poisson form of the propagator, which allows us to write the simplified expressions

$$
\begin{gathered}
G_{0}(u)=\sum_{a} F(a) e^{-(1-u) T(a+\langle a\rangle)}, \\
G_{1}(u)=\frac{1}{2\langle a\rangle} \sum_{a} F(a)[a+\langle a\rangle] e^{-(1-u) T(a+\langle a\rangle)} .
\end{gathered}
$$

From the self-consistent Eq. (8), setting $\delta=1-u$, and solving at the lowest order in $\delta>0$, we find, close to the transition,

$$
\delta \simeq \frac{2 \kappa_{1}}{\kappa_{3} T^{2}}\left(\frac{T-T_{p}^{0}}{T_{p}^{0}}\right),
$$

recovering the Molloy Reed criterion, Eq. (12), for the onset of the giant component. Since the derivatives of $G_{0}(u)$ are finite, we can obtain the size of the giant component $S$ by expanding Eq. (13) close to $u=1$,

$$
\begin{aligned}
S & \simeq 1-G_{0}(1)+\delta G_{0}^{\prime}(1)-\frac{\delta^{2}}{2} G_{0}^{\prime \prime}(1) \\
& =\frac{2 \kappa_{1}^{2}}{\kappa_{3} T}\left(\frac{T-T_{p}^{0}}{T_{p}^{0}}\right)-\frac{2 \kappa_{2} \kappa_{1}^{2}}{\kappa_{3}^{2} T^{2}}\left(\frac{T-T_{p}^{0}}{T_{p}^{0}}\right)^{2} .
\end{aligned}
$$

Since Eq. (16) is obtained from a Taylor expansion for $\delta \ll 1$, we expect it to be valid only close to the percolation threshold.

In order to check the validity of the analytical results developed above, we consider the concrete case of two different forms of activity distribution, namely a uniform activity distribution $F(a)=a_{\max }^{-1}$, with $a \in\left[0, a_{\max }\right]$, and the empirically observed case of power-law activity distribution in social networks [8], $F(a) \simeq(\gamma-1) \epsilon^{\gamma-1} a^{-\gamma}, a \in[\epsilon, 1]$, where $\epsilon$ is the minimum activity in the system. In this last case, we note that the analytical form of the activity distribution is valid for small $\epsilon$ only in the limit of large $N$. Indeed, a simple extreme value theory calculation [34] shows that, in a random sample of $N$ values $a_{i}$, the maximum activity scales as $\min \left\{1, \varepsilon N^{1 /(\gamma-1)}\right\}$. Therefore, when performing numerical simulations of the model, one must consider systems sizes with $N>N_{c}=\epsilon^{1-\gamma}$ in order to avoid additional finite-size effects. In the case of performing simulations for system with small sizes $N<N_{c}$, for example to study the finite-size effects on the percolation threshold (see next section), we used a deterministic power law distribution to avoid the cutoff effect on the maximum value of the activity due to a random sampling of values.

Figure 1 shows the giant component size $S$ of the activity driven network as a result of numerical simulations for both uniform and power-law activity distributions. In Fig. 1(a) we compare $S$ with the analytical approximation Eq. (16), as well as with the result of a direct numerical integration of Eqs. (7) and (8), for uniform activity and power-law activity with exponent $\gamma>3$. In both cases we observe an almost exact match between numerical simulations and the numerical integration of the generating function equations, and a very good agreement with the analytical approximation in the vicinity of the percolation threshold. Figure 1(b), on the other hand, focuses on power-law activity distributions with an exponent smaller than 3 . In this case we additionally plot a

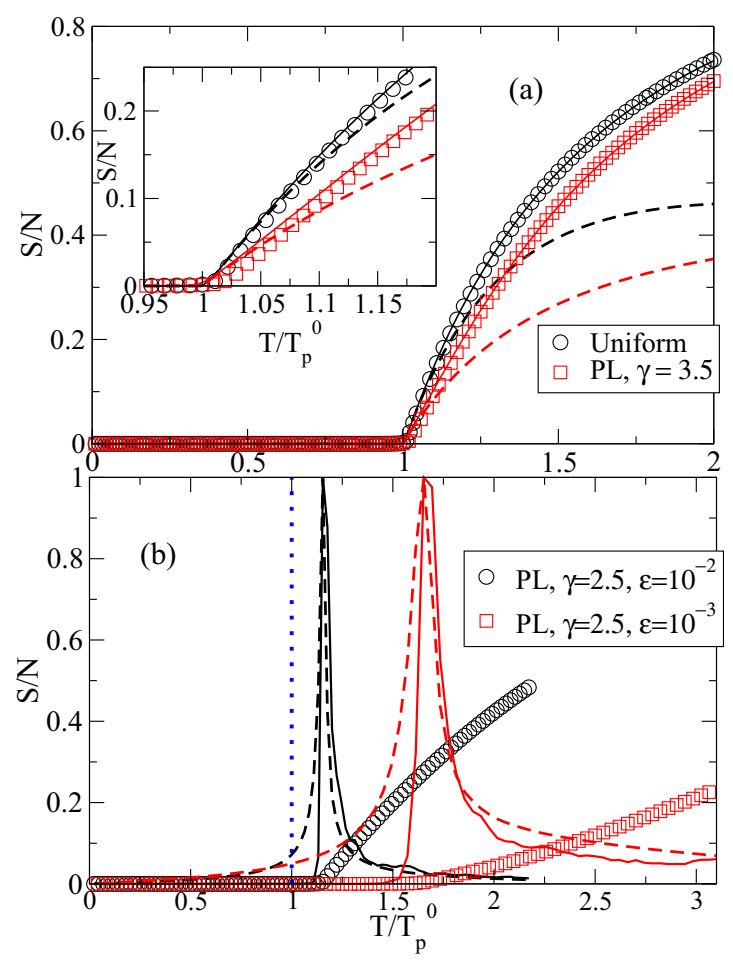

FIG. 1. (Color online) Rescaled giant component size $S / N$ as a function of the rescaled time $T / T_{p}^{0}$ for activity-driven networks. (a) Uniform $\left(a_{\max }=0.01\right)$ and power-law $(\gamma=3.5, \epsilon=0.01)$ activity distributions, compared with the numerical integration of the generating function equations (continuous line) and the theoretical approximation Eq. (16) (dashed line). The inset shows the details close to the percolation threshold $T_{p}$. (b) Power-law activity distribution with $\gamma=2.5, \epsilon=10^{-2}$, and $\epsilon=10^{-3}$. The peaks of the variance of the giant component size, $\sigma(S)^{2}$, and the susceptibility of the cluster size, $\chi(s)$, are plotted with continuous and dashed lines, respectively. Percolation threshold $T_{p}$ is plotted with the dotted line for reference. Results are averaged over $10^{2}$ runs, network size $N=10^{7}$.

numerical estimation of the percolation threshold as given by the peak of both the variance of the giant connected component size $S, \sigma(S)^{2}=\left\langle S^{2}\right\rangle-\langle S\rangle^{2}$, and the susceptibility of the cluster size, $\chi(s)=\sum_{s=2}^{S-1} s^{2} n_{s}$, where $n_{s}$ is the number of clusters of size $s$. From this figure we can see that the numerical percolation threshold strongly deviates in this case from the theoretical prediction Eq. (12), deviation that increases when the distribution cutoff $\varepsilon$ becomes smaller.

\section{EFFECT OF DEGREE CORRELATIONS ON THE TEMPORAL PERCOLATION THRESHOLD}

The origin of the disagreement for the case of powerlaw activity distribution with $\gamma<3$ can be traced back to the effect of degree correlations in the integrated networks generated by the activity-driven model. Indeed, as stated above, the generating function technique makes the explicit assumption of lack of degree correlations [31]. However, the integrated activity-driven network has been shown [28] to exhibit degree correlations, as measured by an average degree 
of the neighbors of the vertices of degree $k, \bar{k}_{T}^{n n}(k)$ [35], being a decreasing function of $k$. Another, global measure of degree correlations can be defined in terms of the Pearson correlation coefficient $r$ between the degree of a node and the mean degree of its neighbors [36], taking the form

$$
r=\frac{\langle k\rangle \sum_{k} k^{2} \bar{k}^{n n}(k) P(k)-\left\langle k^{2}\right\rangle^{2}}{\langle k\rangle\left\langle k^{3}\right\rangle-\left\langle k^{2}\right\rangle^{2}} .
$$

We can easily evaluate the sum $\sum_{k} k^{2} \bar{k}_{T}^{n n}(k) P_{T}(k)$ by applying the hidden variable formalism presented in Sec. II. Inserting in Eq. (17) the first moments of the degree distribution as obtained from Eq. (10), the coefficient $r$ in the limit of large $N$ reads

$$
r_{T}=-\frac{\left(\sigma_{a}^{2}\right)^{2}}{\frac{\kappa_{1} \kappa_{2}}{T}+\kappa_{1} \kappa_{3}-\kappa_{2}^{2}},
$$

where $\sigma_{a}^{2}=\left\langle a^{2}\right\rangle-\langle a\rangle^{2}$ is the variance of the activity distribution. Both the decreasing functional form of $\bar{k}_{T}^{n n}(k)$ and the negative value of $r$ (since $\kappa_{1} \kappa_{3}>\kappa_{2}^{2}$ for any probability distribution with a positive support) indicate the presence of disassortative correlations [36] in the integrated activity-driven networks, correlations whose amplitude is modulated by $\sigma_{a}^{2}$.

In order to take into account the effect of degree correlations let us consider the general problem of percolation in a correlated random network [37]. The effects of the degree correlations are accounted by the branching matrix

$$
B_{k k^{\prime}}=\left(k^{\prime}-1\right) P\left(k^{\prime} \mid k\right),
$$

where $P\left(k^{\prime} \mid k\right)$ is the conditional probability that a node with degree $k$ is connected to a node with degree $k^{\prime}$ [35]. The percolation threshold is determined by the largest eigenvalue $\Lambda_{1}$ of the branching matrix $B_{k k^{\prime}}$ through the condition $\Lambda_{1}=1$. If the network is uncorrelated, $\Lambda_{1}$ reduces to the ratio of the first two moments of the degree distribution, $\Lambda_{1}^{0}=\left\langle k^{2}\right\rangle /\langle k\rangle-1$, thus recovering the Molloy-Reed criterion Eq. (9).

In activity-driven networks we can compute the largest eigenvalue $\Lambda_{1}$ in the limit of small $\lambda$ by applying the hidden variables mapping from Sec. II. In fact, the conditional probability $P_{T}\left(k^{\prime} \mid k\right)$ of the integrated network at time $T$ can be written as [27]

$$
\begin{aligned}
P_{T}\left(k^{\prime} \mid k\right)= & \frac{N}{P_{T}(k)} \sum_{a, a^{\prime}} g_{T}\left(k-1 \mid a^{\prime}\right) F\left(a^{\prime}\right) \frac{\Pi_{T}\left(a^{\prime}, a\right)}{\bar{k}_{T}(a)} \\
& \times F(a) g_{T}(k \mid a),
\end{aligned}
$$

where $\bar{k}_{T}(a)=N \sum_{a} F(a) \Pi_{T}\left(a, a^{\prime}\right)$ [28]. From here, the branching matrix takes the form

$$
B_{k k^{\prime}}=\left(k^{\prime}-1\right)\left[p_{k^{\prime}-1}+\frac{p_{k-1}}{k p_{k}}\left(k^{\prime} p_{k^{\prime}}-\langle k\rangle p_{k^{\prime}-1}\right)\right],
$$

where we write $P_{T}(k)$ as $p_{k}$ for brevity. Assuming that the branching matrix is irreducible, and given that it is nonnegative [see Eq. (19)], we can compute its largest eigenvalue by applying Perron-Frobenius theorem [38] and looking for a principal eigenvector $v_{k}$ with positive components. Using the ansatz $v_{k}=1+\alpha p_{k-1} / k p_{k}$, we obtain that, in order to be an eigenvector, the following conditions must be fulfilled:

$$
\begin{aligned}
\Lambda_{1} & =\langle k\rangle_{T}+\alpha \sum_{k} \frac{(k-1) p_{k-1}^{2}}{k p_{k}}, \\
\Lambda_{1} \alpha & =\left\langle k^{2}\right\rangle_{T}-\langle k\rangle_{T}-\langle k\rangle_{T}^{2}+\alpha\langle k\rangle_{T}\left(1-\sum_{k} \frac{(k-1) p_{k-1}^{2}}{k p_{k}}\right) .
\end{aligned}
$$

One can see that $\sum_{k}(k-1) p_{k-1}^{2} / k p_{k} \simeq 1$, in the limit of large $N$. Thus we obtain the equation for $\Lambda_{1}$

$$
\Lambda_{1}(T)^{2}-\langle k\rangle_{T} \Lambda_{1}(T)-\left\langle k^{2}\right\rangle_{T}+\langle k\rangle_{T}^{2}+\langle k\rangle_{T}=0 .
$$

By using the form of the moments of the degree distribution given by Eq. (10), we solve Eq. (22). Excluding the nonphysical solution $\Lambda_{1}<0$, one finally finds the largest eigenvalue ${ }^{1}$ of the branching matrix as

$$
\Lambda_{1}(T)=\left(\sqrt{\left\langle a^{2}\right\rangle}+\langle a\rangle\right) T .
$$

From here, the percolation threshold in activity-driven networks follows as

$$
T_{p}=\frac{1}{\sqrt{\left\langle a^{2}\right\rangle}+\langle a\rangle} .
$$

We can understand the results of Fig. 1 by comparing the ratio of the exact threshold $T_{p}$ with the uncorrelated value $T_{p}^{0}$,

$$
Q=\frac{T_{p}-T_{p}^{0}}{T_{p}^{0}}=\frac{\sigma_{a}^{4}}{2\langle a\rangle\left(\sqrt{\left\langle a^{2}\right\rangle}+\langle a\rangle\right)^{3}} .
$$

In the case of a uniform activity distribution, we have $Q=$ $13 / \sqrt{3}-15 / 2 \simeq 5.5 \times 10^{-3}$, and therefore the temporal percolation threshold is given with very good accuracy by the uncorrelated expression. For a power-law activity distribution, the ratio $Q$ depends simultaneously on the exponent $\gamma$ and the minimum activity $\varepsilon$. Thus, for $\gamma<3$, we have that $Q \sim \varepsilon^{(\gamma-3) / 2}$, which diverges for $\varepsilon \rightarrow 0$, indicating a strong departure from the uncorrelated threshold. For $\gamma>3$, on the other hand, $Q$ becomes independent of $\varepsilon$, and it goes to 0 in the limit of large $\gamma$. In the case $\gamma=3.5$ and $\varepsilon=0.01$, for example, we obtain $Q \simeq 1.6 \times 10^{-2}$. This implies an error of less than $2 \%$ in the position of the percolation threshold as given by the uncorrelated expression, explaining the good fit observed in Fig. 1(a).

In Fig. 2 we show the ratio $Q$ as a function of the exponent $\gamma$ of a power-law distributed activity potential for different values of $\epsilon$, computed from numerical simulations by evaluating the percolation threshold from the peak of the variance of the giant component size, $\sigma(S)^{2}$. The numerical result is compared with the analytical prediction given by Eq. (25). In this figure one can see that, although numerical and analytical results are in quite good agreement, they still do not exactly coincide for $\gamma<3$. This is due to the presence of finite-size effects, which have not been taken into account in the percolation theory developed. We can consider the finite-size effects on

\footnotetext{
${ }^{1}$ From Eq. (23) one can find $\alpha=\frac{\sigma_{a}^{2}}{\left(\sqrt{\left\langle a^{2}\right\rangle}+\langle a\rangle\right)} T>0$, confirming the validity of the proposed ansatz for $v_{k}$.
} 


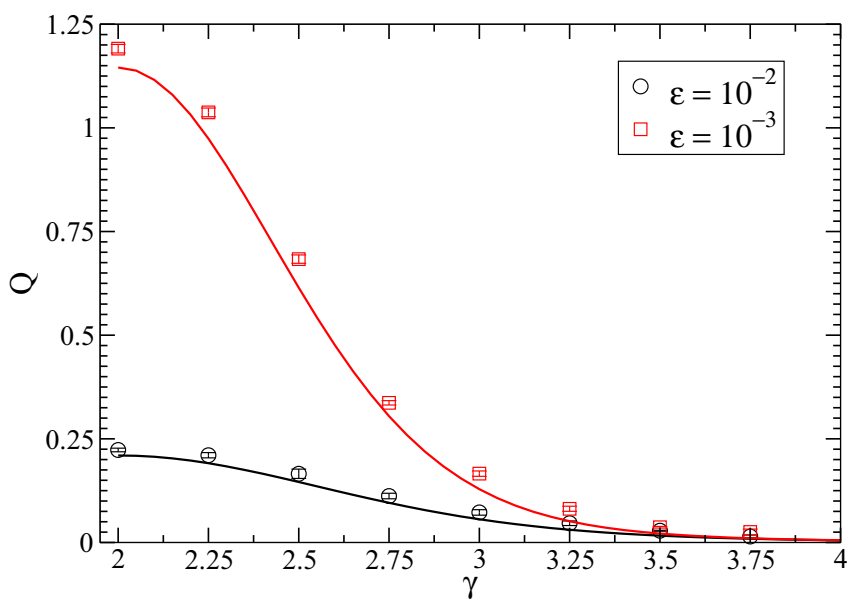

FIG. 2. (Color online) Ratio $Q$, defined in Eq. (25), as a function of the exponent $\gamma$ of the activity potential $F(a) \sim a^{-\gamma}$, for $\epsilon=$ $10^{-2}$ and $\epsilon=10^{-3}$. We compare $Q$ as obtained by estimating the percolation threshold $T_{p}$ from the peak of the variance of the giant component size, $\sigma(S)^{2}$, by means of a numerical simulation of a network with size $N=10^{7}$ (symbols), with the prediction of Eq. (25) (lines). The results of numerical simulations are averaged over $10^{2}$ runs.

the percolation time $T_{p}(N)$ in a network of size $N$ by putting forward the standard hypothesis of a scaling law of the form

$$
T_{p}(N)=T_{p}+A N^{-v} .
$$

In Fig. 3 we plot the rescaled numerical thresholds $\left[T_{p}(N)-\right.$ $\left.T_{p}\right] / T_{p}$ estimated by the peak of the variance of the giant component size, $\sigma(S)^{2}$, as a function of the network size $N$. We can observe that the numerical thresholds $T_{p}(N)$ asymptotically

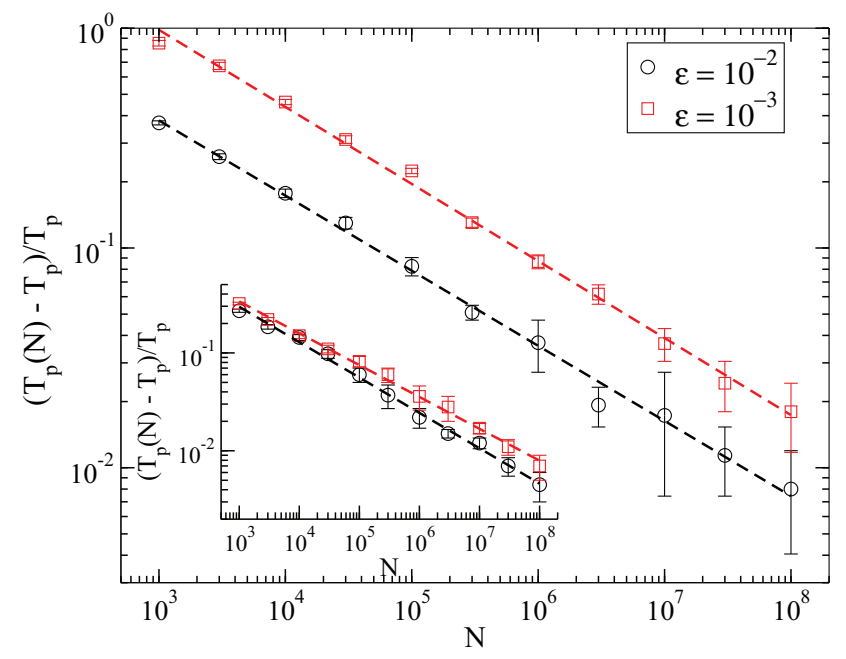

FIG. 3. (Color online) Finite-size scaling of the percolation threshold as estimated by the peak of the variance of the giant component size, $\sigma(S)^{2}$, for a network with power-law activity distribution with $\gamma=2.5$ (main) and $\gamma=3.5$ for different values of $\epsilon$. We plot $\left[T_{p}(N)-T_{p}\right] / T_{p}$ as a function of $N$, finding a scaling of the form of Eq. (26), plotted with dashed lines, with the same exponent $v=0.34 \pm 0.01$ for $\gamma=2.5$ and $v=0.34 \pm 0.02$ for $\gamma=3.5$. The results are averaged over $10^{2}$ runs. tend to the theoretical prediction $T_{p}$ by following the scaling law of Eq. (26), with a very similar exponent $v \simeq 0.34 \pm 0.02$, and different values of the prefactor $A$ depending on the values of $\gamma$ and $\epsilon$. We also checked (data not shown) that the other method to measure the percolation threshold, through the susceptibility of the clusters size $\chi(s)$, follows the same scaling law, with the same exponent $v$ and a slightly different prefactor $A$.

\section{APPLICATION TO EPIDEMIC SPREADING}

The concept of temporal percolation can be applied to gain understanding of epidemic processes on activity-driven temporal networks [8]. Let us focus on the susceptibleinfected-removed (SIR) model [30], which is the simplest model representing a disease that confers immunity and that is defined as follows: Individuals can be in either of three states, namely susceptible, infected, or removed. Susceptible individuals acquire the disease by contact with infected individuals, while infected individuals heal spontaneously becoming removed, which cannot contract the disease anymore. On a temporal network, the SIR model is parametrized by the rate $\mu$ (probability per unit time) at which infected individuals become removed, and by the transmission probability $\beta$ that the infection is propagated from an infected individual to a susceptible individual by means of an instantaneous contact.

We can approach the behavior of the SIR model on activitydriven networks by extending the mapping to percolation developed in Ref. [29] into the temporal case. To do so, let us consider first a modified SIR model in which individuals stay in the infected state for a fixed amount of time $\tau$. We define the transmissibility $\mathcal{T}_{i j}$ as the probability that the infection is transmitted from infected individual $i$ to susceptible individual $j$. Considering that contacts last for an amount of time $\Delta t=1 / N$, the transmissibility can be written as

$$
\mathcal{T}_{i j}(\beta, \tau)=1-\left(1-\beta p_{i j}\right)^{\tau N},
$$

where $p_{i j}=\left(a_{i}+a_{j}\right) / N^{2}$ is the probability that individuals $i$ and $j$ establish a contact in any given time step $\Delta t$ [28]. In the limit of large $N$, we can thus write

$$
\mathcal{T}_{i j}(\beta, \tau)=1-\exp \left(-\frac{\beta \tau\left[a_{i}+a_{j}\right]}{N}\right) .
$$

From here we can deduce the form of the transmissibility when healing is not deterministic but a Poisson process with rate $\mu$. In this case, the probability that an infected individual remains infected a time $\tau$ is given by the exponential distribution $P(\tau)=\mu e^{-\mu \tau}$. Therefore, we can write [29]

$$
\begin{aligned}
\mathcal{T}_{i j}(\beta, \mu) & =\int_{0}^{\infty} \mathcal{T}_{i j}(\beta, \tau) P(\tau) d \tau \\
& =1-\left(1+\frac{\beta}{\mu} \frac{a_{i}+a_{j}}{N}\right)^{-1} \simeq \frac{\beta}{\mu} \frac{a_{i}+a_{j}}{N}
\end{aligned}
$$

in the limit of large $N$. If we consider the process of infection as equivalent to establishing a link between infected and susceptible individuals and we compare this expression with Eq. (4), we can see that the SIR process can be mapped to the 
creation of the integrated network in the activity-driven model up to a time $T=\beta / \mu$. The epidemic threshold will be given by the existence of a finite cluster of recovered individuals, and therefore will coincide with the temporal percolation threshold; i.e.,

$$
\left(\frac{\beta}{\mu}\right)_{c}=T_{p} .
$$

The temporal percolation threshold given by Eq. (24) recovers the epidemic threshold obtained in Ref. [39] using a mean-field rate equation approach. ${ }^{2}$ A particular benefit of this percolation mapping is the fact that it makes accessible the calculation of explicit approximate forms for the size of epidemic outbreaks, Eq. (16) (valid however in certain limits), which are not easily available in mean-field approximations [8,39].

\section{SUMMARY AND CONCLUSIONS}

In this work we have studied the time evolution of the connectivity properties of the integrated network ensuing from a sequence of pairwise contacts between a set of fixed agents, defining a temporal network. We have focused in particular in the onset of the giant component in the integrated network, defined as the largest set of connected agents that have established at least one contact up to a fixed time $T$. The onset of the giant component takes place at some percolation time $T_{p}$, which depends on the details of temporal network dynamics. Considering in particular the recently proposed activity-driven model [8], and building upon the mapping of this temporal network on a network model with hidden variables [28], we are able to provide analytical expressions for the percolation time. Assuming lack of degree correlations in the initial evolution of the integrated network, the application of the generating

\footnotetext{
${ }^{2}$ Notice that in Ref. [39] the per capita infection rate $\beta^{\prime}=2\langle a\rangle \beta$ is used.
}

function formalism [31] allows us to obtain an explicit general form for the temporal percolation threshold, as well as analytic asymptotic expressions for the size of the giant component in the vicinity of the threshold. These expressions turn out to be in good agreement with numerical results for particular forms of the activity distribution imposing weak degree correlations. For a skewed, power-law distributed activity $F(a) \sim a^{-\gamma}$, the uncorrelated results are still numerically correct for large values of $\gamma$. When $\gamma$ is small, however, strong disagreements arise. Applying a percolation formalism for correlated networks [37], we are able to obtain the analytical threshold $T_{p}$. For $\gamma>3$, the correlated threshold collapses onto the uncorrelated result, which thus provides a very good approximation to the exact result. For small $\gamma<3$, the percolation threshold as obtained by numerical simulation of large networks is in very good agreement with the analytical prediction.

The study of the percolation properties of integrated temporal networks opens in our view new interesting venues of future research, related in particular to the properties of dynamical processes running on top of them and to the coupling of their different time scales. One such application in the context of epidemic spreading is the study of the SIR model, which we have shown can be mapped to a temporal percolation problem in activity-driven networks, thus providing explicit forms (albeit valid in certain limits of weak degree correlations) for the size of epidemic outbreaks in this class of systems. Another interesting application concerns the percolation properties of more realistic variations of the activity-driven model, such as the one proposed in [40].

\section{ACKNOWLEDGMENTS}

We acknowledge financial support from the Spanish MICINN, under Project No. FIS2010-21781-C02-01, and EC FET-Proactive Project MULTIPLEX (Grant No. 317532). R.P.-S. acknowledges additional financial support from ICREA Academia, funded by the Generalitat de Catalunya.
[1] A.-L. Barabási, Nat. Phys. 1, 68 (2005).

[2] M. E. J. Newman, Networks: An Introduction (Oxford University Press, Oxford, 2010).

[3] S. N. Dorogovtsev, A. V. Goltsev, and J. F. F. Mendes, Rev. Mod. Phys. 80, 1275 (2008).

[4] A. Barrat, M. Barthélemy, and A. Vespignani, Dynamical Processes on Complex Networks (Cambridge University Press, Cambridge, 2008).

[5] R. Pastor-Satorras and A. Vespignani, Evolution and Structure of the Internet: A Statistical Physics Approach (Cambridge University Press, Cambridge, 2004).

[6] J.-P. Onnela, J. Saramäki, J. Hyvönen, G. Szabó, D. Lazer, K. Kaski, J. Kertész, and A.-L. Barabási, Proc. Natl. Acad. Sci. USA 104, 7332 (2007).

[7] C. Cattuto, W. Van den Broeck, A. Barrat, V. Colizza, J.-F. Pinton, and A. Vespignani, PLoS ONE 5, e11596 (2010).
[8] N. Perra, B. Gonçalves, R. Pastor-Satorras, and A. Vespignani, Sci. Rep. 2, 469 (2012).

[9] P. Holme and J. Saramäki, Phys. Rep. 519, 97 (2012).

[10] J. G. Oliveira and A.-L. Barabasi, Nature (London) 437, 1251 (2005)

[11] P. Hui, A. Chaintreau, J. Scott, R. Gass, J. Crowcroft, and C. Diot, in WDTN '05: Proceedings of the 2005 ACM SIGCOMM Workshop on Delay-Tolerant Networking (ACM, New York, 2005), pp. 244-251.

[12] P. Holme, Phys. Rev. E 71, 046119 (2005).

[13] J. Tang, S. Scellato, M. Musolesi, C. Mascolo, and V. Latora, Phys. Rev. E 81, 055101 (2010).

[14] L. Isella, J. Stehlé, A. Barrat, C. Cattuto, J.-F. Pinton, and W. V. den Broeck, J. Theor. Biol. 271, 166 (2011).

[15] J. Stehle, N. Voirin, A. Barrat, C. Cattuto, V. Colizza, L. Isella, C. Regis, J.-F. Pinton, N. Khanafer, W. Van den Broeck, and P. Vanhems, BMC Medicine 9, 87 (2011). 
[16] M. Karsai, M. Kivelä, R. K. Pan, K. Kaski, J. Kertész, A.-L. Barabási, and J. Saramäki, Phys. Rev. E 83, 025102 (2011).

[17] G. Miritello, E. Moro, and R. Lara, Phys. Rev. E 83, 045102 (2011).

[18] M. Kivela, R. Kumar Pan, K. Kaski, J. Kertesz, J. Saramaki, and M. Karsai, J. Stat. Mech. (2012) P03005.

[19] R. Parshani, M. Dickison, R. Cohen, H. E. Stanley, and S. Havlin, Europhys. Lett. 90, 38004 (2010).

[20] P. Bajardi, A. Barrat, F. Natale, L. Savini, and V. Colizza, PLoS ONE 6, e19869 (2011).

[21] M. Starnini, A. Machens, C. Cattuto, A. Barrat, and R. PastorSatorras, J. Theor. Biol. 337, 89 (2013).

[22] A. Vazquez, B. Rácz, A. Lukács, and A.-L. Barabási, Phys. Rev. Lett. 98, 158702 (2007).

[23] T. Hoffmann, M. A. Porter, and R. Lambiotte, Phys. Rev. E 86, 046102 (2012).

[24] N. Perra, A. Baronchelli, D. Mocanu, B. Gonçalves, R. PastorSatorras, and A. Vespignani, Phys. Rev. Lett. 109, 238701 (2012).

[25] M. E. J. Newman, Proc. Natl. Acad. Sci. USA 98, 404 (2001).

[26] B. Ribeiro, N. Perra, and A. Baronchelli, Sci. Rep. 3, 3006 (2013).

[27] M. Boguñá and R. Pastor-Satorras, Phys. Rev. E 68, 036112 (2003).
[28] M. Starnini and R. Pastor-Satorras, Phys. Rev. E 87, 062807 (2013).

[29] M. E. J. Newman, Phys. Rev. E 66, 016128 (2002).

[30] R. M. Anderson and R. M. May, Infectious Diseases in Humans (Oxford University Press, Oxford, 1992).

[31] M. E. J. Newman, S. H. Strogatz, and D. J. Watts, Phys. Rev. E 64, 026118 (2001).

[32] M. Molloy and B. Reed, Random Struct. Algorithms 6, 161 (1995).

[33] I. S. Gradshteyn and I. M. Ryzhik, Table of Integrals, Series, and Products, 7th ed. (Elsevier/Academic Press, Amsterdam, 2007).

[34] E. Gumbel, Statistics of Extremes, Dover Books on Mathematics (Dover Publications, New York, 2004).

[35] R. Pastor-Satorras, A. Vázquez, and A. Vespignani, Phys. Rev. Lett. 87, 258701 (2001).

[36] M. E. J. Newman, Phys. Rev. Lett. 89, 208701 (2002).

[37] A. V. Goltsev, S. N. Dorogovtsev, and J. F. F. Mendes, Phys. Rev. E 78, 051105 (2008).

[38] F. R. Gantmacher, The Theory of Matrices, Vol. II (Chelsea Publishing Company, New York, 1959).

[39] S. Liu, N. Perra, M. Karsai, and A. Vespignani, arXiv:1309.7031.

[40] M. Karsai, N. Perra, and A. Vespignani, Sci. Rep. 4, 4001 (2014). 